\title{
Effect of Occlusal Reduction on Postoperative Pain After Endodontic Treatment in Teeth with Irreversible pulpitis and Symptomatic Apical Periodontitis: A Randomized Clinical Trial
}

\section{Marian Shenoda Fathy ${ }^{1 *}$, Hebatallah Mohamed Maged ELFar ${ }^{2}$ and Shaimaa Ismail Gawdat ${ }^{3}$}

${ }^{1}$ Master Degree in Endodontics, Oral and Dental Medicine, Cairo University, Giza, Egypt

${ }^{2}$ Professor of Endodontics, Faculty of Dentistry, Cairo University, Giza, Egypt

${ }^{3}$ Assistant Professor of Endodontics, Faculty of Dentistry, Cairo University, Giza,

Egypt

*Corresponding Author: Marian Shenoda Fathy, Master Degree in Endodontics, Oral and Dental Medicine, Cairo University, Giza, Egypt.
Received: June 28, 2021

Published: August 07, 2021

(C) All rights are reserved by Marian Shenoda Fathy., et al.

\begin{abstract}
Introduction: Evaluation of pain intensity after endodontic instrumentation and obturation after occlusal reduction in mandibular posterior teeth associated with symptomatic irreversible pulpitis and symptomatic apical periodontitis.

Aim of the Study: To assess the effect of occlusal reduction on post-operative pain after endodontic instrumentation and obturation in patients with symptomatic irreversible pulpitis and symptomatic apical periodontitis in mandibular posterior teeth in a randomized clinical trial.

Methods: Forty-four patients diagnosed with symptomatic irreversible pulpitis and symptomatic apical periodontitis in mandibular posterior teeth were included in this trial. Participants were randomly allocated into two equal groups. In the group A, all occlusal contacts on the functional and non-functional cusps as well as on the marginal ridges of the teeth were reduced by $2 \mathrm{~mm}$, while in group B, no modification was done. Canal instrumentation was completed on the first visit using Revo-S rotary files. Post-instrumentation pain intensity was assessed at 6,12,24 and $48 \mathrm{hrs}$ using visual analogue. Canal obturation was completed 7days later and pain was recorded at 12 and 24 hours. In case of severe postoperative pain, analgesics and placebo were prescribed to the participants.
\end{abstract}

All demographic data, post obturation radiographic findings, VAS scores and analgesic intake were statistically analyzed.

Result: Results showed that occlusal reduction has no significant difference in pain reduction at 6 hours, 12 hours, 24 hours, and 48 hours post-instrumentation and at 6 hours and 12 hours post-obturation. There was a decrease in the median VAS score through the follow up periods in the two groups with a maximum decrease at 48 hours following root canals instrumentation. There was no significant difference in incidence of analgesic intake between the two groups.

Conclusion: Within the limitations of this study, we conclude that occlusal reduction in teeth with irreversible pulpitis and with tenderness to percussion had no significant influence on postoperative pain after root canal preparation when compared with teeth with no occlusal reduction. Pain intensity gradually decreases by time following endodontic treatment.

Keywords: Apical Periodontitis; Occlusal Reduction; Pulpitis; Postoperative Pain; Randomized Clinical Trial

Citation: Marian Shenoda Fathy., et al. "Effect of Occlusal Reduction on Postoperative Pain After Endodontic Treatment in Teeth with Irreversible pulpitis and Symptomatic Apical Periodontitis: A Randomized Clinical Trial". Acta Scientific Dental Sciences 5.9 (2021): 46-54. 


\section{Introduction}

Postoperative pain is defined as pain of any degree that occurs after initiation of root canal treatment (RCT). The incidence of postoperative pain was reported to range from $3 \%$ to $58 \%$ [1]. Development of postoperative pain after RCT is usually due to acute inflammatory response in the periapical tissues. It commences within a few hours or days after endodontic treatment $[2,3]$.

Several strategies have been described for managing pain and discomfort after RCT. These include preoperative analgesics and corticosteroid prescription [4-6], occlusal reduction [7,8] and administration of long-acting anesthesia [9-11].

After searching on PubMed database for evidence-based investigations on evaluating the effect of occlusal reduction on pain after endodontic treatment, it was found that some authors [7,12,13] reported a significant effect of occlusal reduction on reducing postoperative pain. However, others [11,14-19] reported no significant differences in postoperative pain and discomfort in patients who had received RCT with or without occlusal reduction.

Different results from such studies, were due to many factors; the differences in inclusion criteria, number of treatment visits, the gender and age of the patients, presence of preoperative pain, tenderness to percussion before RCT [15], type of intracanal medication used, incomplete removal of pulp tissues, overextension of root canal filling materials and sealers [20] or apical extrusion of infected debris during chemomechanical instrumentation, which may generate an acute inflammatory response to the periapical tissue $[6,21]$.

Therefore, this study was to evaluate the effect of occlusal reduction on pain after endodontic instrumentation and obturation in patients with symptomatic irreversible pulpitis and symptomatic apical periodontitis.

\section{Materials and Methods}

Trial design

The trial design of this study was a parallel, randomized, double blinded and controlled clinical trial. Randomized clinical trials are the gold standard of clinical research applied to new medical interventions.

\section{Ethical consideration}

The protocol of the trial was approved by the Ethics committee, Faculty of Oral and Dental Medicine, Cairo University. Each patient was informed to follow general instructions and sign a printed consent explaining the aim of the study, the nature of the procedure and possible discomforts. The patients were obligated to fill the pain scale chart daily postoperatively at 6,12, 24 and 48 hours after root canal instrumentation and at 6 and 12 hours after root canals obturation accurately and honestly. The patients were instructed to return it to the operator at the specific time.

\section{Participant}

Sample size

The study was planned with 20 participants in the control group and 20 participants in the intervention group, based on the probability of Type 1 error 0.05 and power at 0.8 . The expected dropout rate in this experiment was $10 \%$; hence the total sample size was 22 interventional subjects and 22 control subjects.

\section{Eligibility criteria}

The inclusion criteria for participants were healthy adults (ASA I) of 18 - 50 years complaining of moderate or severe sharp throbbing pain related to a posterior mandibular tooth. Included teeth had moderate or severe preoperative pain that occurred spontaneously or provoked by cold and/or hot stimulus and lingered after removal of the stimulus with pain on biting or chewing. The diagnosis of symptomatic irreversible pulpitis with symptomatic apical periodontitis was confirmed by the history of the chief complaint, clinical examination revealing positive response to an electrical pulp tester (Denjoy DY310 Dental Pulp Tester, Denjoy, Henan, China), tenderness to percussion on tapping the tooth with the end of a mirror handle and radiographic examination revealing no apical radiolucency or only slight widening of the periodontal membrane space. The contralateral tooth was used as a control.

The case was excluded if the offending tooth had no occlusal contact, premature contact, was associated with acute or chronic periapical abscess, had fewer than three teeth on one side of the jaw, had greater than grade I mobility or pocket depth greater than $5 \mathrm{~mm}$ and was not restorable, or the patient was a pregnant woman or classified other than ASA I. Moreover, patients were excluded if they reported bruxism or clenching, and if analgesics or nonsteroi- 
Effect of Occlusal Reduction on Postoperative Pain After Endodontic Treatment in Teeth with Irreversible pulpitis and Symptomatic Apical Periodontitis: A Randomized Clinical Trial

dal anti-inflammatory drugs had been administered during the 12 $\mathrm{h}$ preoperatively that might alter their pain perception.

\section{Setting and location}

All patients were selected and treated in the Endodontic clinic of Faculty of Dentistry, Cairo University.

\section{Randomization}

Forty-four numbers were generated by the center of EvidenceBased Dentistry, Faculty of Oral and Dental Medicine, Cairo University and randomly allocated to either intervention or control group in Excel (Microsoft Office Excel 2010; Microsoft Corporation, Redmond, WA, USA) using block randomization and printed on a table. Patients were given numbers sequentially after confirming eligibility. To ensure allocation concealment, the table was kept and only accessed by a co-investigator who was contacted to reveal the assigned group of each patient.

\section{Endodontic procedure}

The endodontic treatment of all cases was done in two visits. Before treatment, each patient was asked to record his/her pain level before any endodontic treatment. Patients with moderate (4 - 6) to severe (7 - 10) preoperative pain were included. The tooth was anesthetized using inferior alveolar nerve block technique by local anesthesia of 2\% Mepivacaine $\mathrm{HCl}$ (Mepecaine-L, Alexandria Company for Pharmaceuticals and Chemical Industries, Alexandria, Egypt) with 1:100,000 epinephrine, one or two carpules (1.8 - $3.6 \mathrm{~mm}$ ) according to severity of pain. Supplemental anesthesia of choice was intra-pulpal if there was persistent pain. The access cavity preparation was performed using round carbide bur and Endo-Z bur. After access cavity preparation, patient allocation was undertaken.

In the intervention group; all occlusal contacts on the functional and non-functional cusps as well as on the marginal ridges were reduced to be free contact with opposing teeth using a diamond bur in a high-speed handpiece with copious water spray. An articulating paper was used to confirm free occlusal contacts. While in control group; all occlusal contacts were left intact. The tooth was properly isolated with a rubber dam. The patency of the root canals was confirmed using stainless steel hand K-files size \#10 and \#15 (K-files; Mani, Inc., Tochigi, Japan). Working length was determined using an electronic apex locator then confirmed with intraoral peri- apical (Root ZX, J. Morita USA, Irvine, CA, USA) and radiographically, to be $0.5-1 \mathrm{~mm}$, shorter than radiographic apex using the bisecting angle technique.

Mechanical preparation of root-canals was done by crown-down technique using rotary Revo-S (Revo-S; Micro-Mega, Besançon, France) instruments in an endodontic motor (X-Smart; Dentsply Sirona, Ballaigues, Switzerland) at a speed of $300 \mathrm{rpm}$, and the torque was adjusted according to the manufacturer instructions.

The mesial root canals, distal root with two canals in molars, and premolars with more than one root canal were prepared till AS 35 or AS 30 in severely curved canals till the corresponding manual file is fitting snugly at the apex. The distal root in molars with one canal and premolars with one canal were prepared till AS 40. In situations where root canals were too large for available sizes of Revo-S files, where the initial file was larger than size 25, mechanical preparation of the apical third was completed using manual stainless steel K files sizes 45 - 55.

The canals were thoroughly irrigated using $3 \mathrm{ml}$ of 1:1 diluted of Sodium hypochlorite concentration $2.6 \%$ between every two subsequent instruments using a plastic disposable syringe ( $3 \mathrm{ml}$ ) with needle gauge 27. EDTA gel was used as a lubricant. Master cone selection with (0.04) taper was selected corresponding to the same size as the master apical file and confirmed by periapical radiograph using the bisecting angle technique.

After dryness, a cotton pellet was placed in the pulp chamber and the access cavity was closed with a temporary filling (MDTemp; Meta Biomed Co., Ltd, Chungbuk, Korea).

For post-instrumentation pain assessment after instrumentation: Patients were given two capsules (placebo and Ibuprofen 400 $\mathrm{mg}$ ). Placebo is a pharmacologically inert substance with no therapeutic effect. It acts by controlling anxiety and it has been shown by many studies to be effective in high percentage of patients 46 . Patients took placebo in a form of opaque capsules filled with powdered milk. Ibuprofen was prescribed in case of persistent pain.

Participants were recalled after 7 days from the first visit for completion of root canal treatment. The rubber dam was placed, temporary filling was removed using high speed handpiece and reirrigation of root canals was done with the same technique as the first visit. The canals were dried using paper points and obturated 
with previously selected master cone. Obturation was done using modified single cone technique and AdSeal (AdSeal; Meta Biomed Co. Ltd). resin-based root canal sealer with the help of spreader size 30 and Gutta percha auxiliaries size 25. After obturation, the access cavity was cleaned from excess sealer with cotton and sealed with a temporary filling. Finally, a periapical radiograph was taken to check obturation.

\section{Outcomes assessment}

The primary outcome was the degree of postoperative pain, which was measured using a visual analogue scale (VAS) after 6, 12,24 and 48 hours from the end of endodontic instrumentation then after 6 and 12 hours from the end of root canal obturation.

The scale was numerical, visual and verbal to facilitate its use by the participants. The patients were asked to choose the mark that represented their level of pain. Pain level was assigned to one of 4 categorical scores: 1, None (0); 2, Mild (1 - 3); 3, Moderate (4 - 6); 4, Severe $(7-10)$. The operator kept in contact with the participants by phone, to remind them and to ensure accurate adherence to instructions.

Secondary outcome was the number and the time of placebo and analgesic tablets taken by the patient. The patients were instructed to take only one capsule of placebo in case of presence of moderate $(4-6)$ or severe $(7-10)$ postoperative pain. In case of persistent moderate or severe pain, patients were allowed to take Ibuprofen (400 mg) and instructed to record the number of administrated Ibuprofen tablets.

\section{Statistical analysis}

Mann-Whitney (U) and Independent t (T) tests were used to compare between groups. Chi-square $\left(\chi^{2}\right)$ and Fisher Exact $(F)$ tests were used for categorical data. Friedman test followed by Wilcoxon signed rank test with Bonferroni correction was used to compare VAS scores within groups between follow up periods. Statistical calculation was undertaken using the SPSS package for Windows (2006; Statistical Package for the Social Science; SPSS Inc., Chicago, IL, USA). The significance level was set at $\mathrm{P} \leq 0.05$.

\section{Results}

From 54 enrolled patients, 44 patients were included in the study. The flow chart of the patients through the study following the CONSORT flow diagram is presented in figure 1.

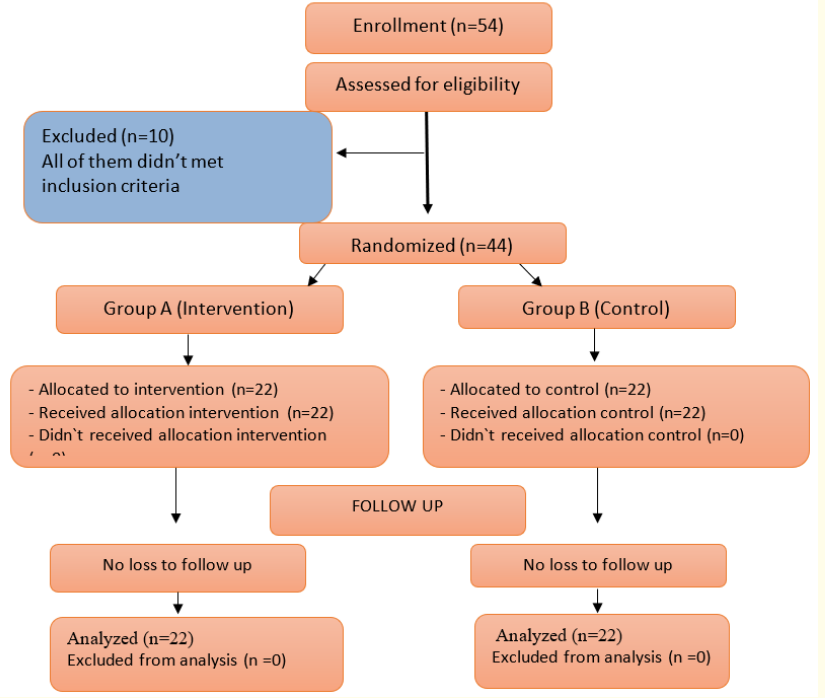

Figure 1: CONSORT flow diagram of the study.

\section{Demographic data}

There was no significant difference in the baseline data of mean age values, gender distribution, tooth type distribution or number of root canals between the two groups $(\mathrm{P}>0.05)$ as mentioned in table 1.

\begin{tabular}{|l|c|c|c|c|c|c|}
\hline \multicolumn{2}{|c|}{ Group Variables } & \multicolumn{2}{|c|}{ Group A } & \multicolumn{2}{c|}{ Group B } & \multirow{2}{*}{ P value } \\
\cline { 2 - 7 } \multicolumn{2}{|c|}{ No } & $\%$ & No & $\%$ & & \\
\hline \multirow{2}{*}{ Age (yrs.) } & Mean \pm SD & \multicolumn{2}{|c|}{$34.8 \pm 7.7$} & \multicolumn{2}{|c|}{$33.9 \pm 9.8$} & 0.734 \\
\cline { 2 - 7 } & Range & $23-50$ & \multicolumn{2}{|c}{$18-49$} & \\
\hline \multirow{2}{*}{ Sex } & Female & 14 & 63.6 & 15 & 68.2 & 0.750 \\
\cline { 2 - 7 } & Male & 8 & 36.4 & 7 & 31.8 & \\
\hline \multirow{2}{*}{$\begin{array}{l}\text { Tooth } \\
\text { type }\end{array}$} & Premolar & 9 & 40.9 & 10 & 45.5 & 0.761 \\
\cline { 2 - 7 } & Molar & 13 & 59.1 & 12 & 54.5 & \\
\hline
\end{tabular}

Table 1: Descriptive statistics, independent t-test and Chi square tests for the demographic data of the tested groups (Group A; occlusal reduction, Group B: no occlusal reduction). *: Statistically significant ( $\mathrm{p}$-value $\leq 0.05$ ).

Primary outcome

Incidence of pre-operative and post-instrumentation pain

Both groups showed pre-operative severe pain in all patients. There was no statistically significant difference in pain incidence at 
Effect of Occlusal Reduction on Postoperative Pain After Endodontic Treatment in Teeth with Irreversible pulpitis and Symptomatic Apical Periodontitis: A Randomized Clinical Trial

different pain categories between the two groups using Chi square test as mentioned in figure 2 and 3.

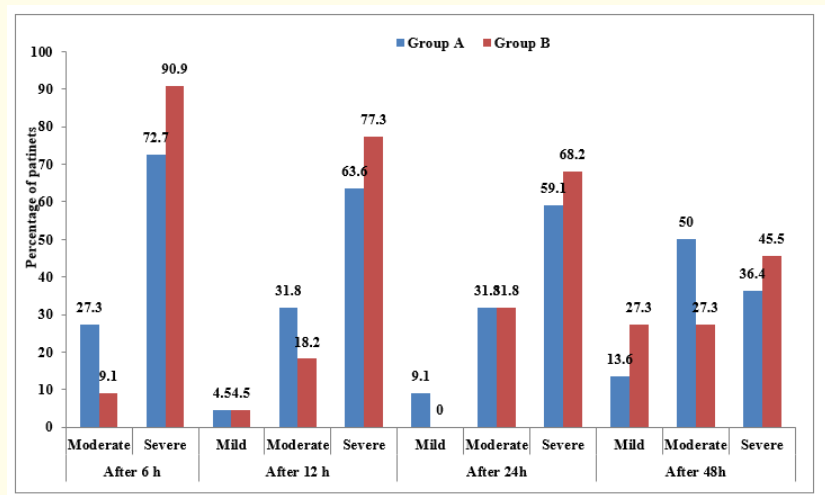

Figure 2: Bar chart showing incidence of different categories of preoperative and post-instrumentation pain in the two groups. (Group A; occlusal reduction, Group B: no occlusal reduction).

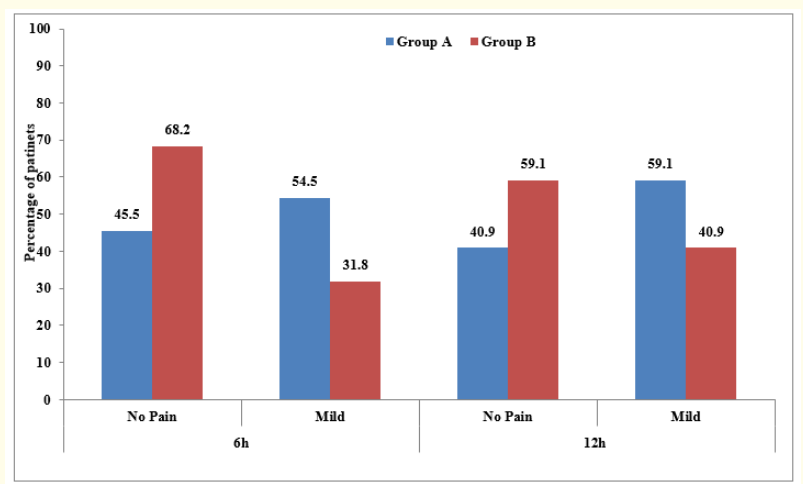

Figure 3: Bar chart showing incidence of different categories of post-obturation pain in the two groups (Group A; occlusal reduction, Group B: no occlusal reduction).

Comparison of pain intensity of pre-operative and post-instrumentation pain at different follow-up periods between the two groups

There was no statistically significant difference in the median and range values of MVAS scores between both groups as mentioned in table 2.

\begin{tabular}{|c|c|c|c|c|c|c|c|}
\hline \multirow{2}{*}{$\begin{array}{c}\text { Groups } \\
\text { different } \\
\text { times }\end{array}$} & \multicolumn{3}{|c|}{$\begin{array}{l}\text { Group A (occlusal } \\
\text { reduction) }\end{array}$} & \multicolumn{3}{|c|}{$\begin{array}{c}\text { Group B (No } \\
\text { occlusal reduction) }\end{array}$} & \multirow{2}{*}{$\begin{array}{c}\text { P value } \\
1\end{array}$} \\
\hline & Median & Min. & Max. & Median & Min. & Max & \\
\hline $\begin{array}{l}\text { Preoperative } \\
\text { pain }\end{array}$ & 9 & 7 & 10 & 9 & 8 & 10 & 0.248 \\
\hline 6 Hours & 8 & 4 & 9 & 9 & 6 & 9 & 0.088 \\
\hline 12 Hours & 7 & 3 & 9 & 8 & 3 & 10 & 0.176 \\
\hline 24 Hours & 7 & 3 & 9 & 7 & 4 & 9 & 0.513 \\
\hline 48 Hours & 5 & 2 & 9 & 5 & 2 & 9 & 0.704 \\
\hline P value & \multicolumn{3}{|c|}{$<0.001$} & \multicolumn{3}{|c|}{$<0.001$} & \\
\hline 6 hours & 1 & 0 & 2 & 0 & 0 & 3 & 0.564 \\
\hline 12 hours & 1 & 0 & 2 & 0 & 0 & 3 & 0.689 \\
\hline P value & \multicolumn{3}{|c|}{0.655} & \multicolumn{3}{|c|}{1.000} & \\
\hline
\end{tabular}

Table 2: Median and range of MVAS score at different time points in the two groups by Mann Whitney test.

*: Statistically significant ( $\mathrm{p}$-value $\leq 0.05$ ).

Changes with time in pain intensity within each group

There was statistically significant decrease in median VAS scores comparing preoperative with $12 \mathrm{~h}, 24 \mathrm{~h}$ and $48 \mathrm{~h}$ in the two groups. There was statistically significant decrease in median VAS scores comparing $6 \mathrm{~h}$ with $48 \mathrm{~h}$. However, there was no statistically significant decrease in median VAS scores comparing all other time periods as mentioned in table 3 .

\begin{tabular}{|l|c|c|}
\hline & $\begin{array}{c}\text { P value in } \\
\text { group A }\end{array}$ & $\begin{array}{c}\text { P value in } \\
\text { group B }\end{array}$ \\
\hline After 6h vs. Preoperative & 0.076 & 0.701 \\
\hline After 12 Hours - Preoperative & 0.001 & 0.004 \\
\hline After 24 Hours vs. Preoperative & $<0.001$ & $<0.001$ \\
\hline After 48 Hours vs. Preoperative & $<0.001$ & $<0.001$ \\
\hline After 12 Hours vs. After 6h & 1.000 & 0.861 \\
\hline After 24 Hours vs. After 6h & 0.150 & 0.004 \\
\hline After 48 Hours vs. After 6h & 0.001 & 0.012 \\
\hline After 24 Hours vs. After 12 Hours & 1.000 & 0.701 \\
\hline After 48 Hours vs. After 12 Hours & 0.076 & 1.000 \\
\hline After 48 Hours vs. After 24 Hours & 1.000 & 1.000 \\
\hline
\end{tabular}

Table 3: Pairwise comparison by Wilcoxon signed rank test in VAS scores by time.

*: Statistically significant ( $p$-value $\leq 0.05)$. 
Effect of Occlusal Reduction on Postoperative Pain After Endodontic Treatment in Teeth with Irreversible pulpitis and Symptomatic Apical Periodontitis: A Randomized Clinical Trial

\section{Secondary outcome}

There was no significant difference in the incidence of placebo and analgesic intake and number of analgesic tablets between the two groups as mentioned in table 4.

\begin{tabular}{|l|c|c|c|c|c|c|}
\hline \multicolumn{2}{|l|}{ Post Instrumentation Records } \\
\hline \multirow{2}{*}{ Group Variables } & \multicolumn{2}{|c|}{ Group A } & \multicolumn{2}{|c|}{ Group B } & \\
\cline { 2 - 7 } No & $\%$ & No & $\%$ & P value & \\
\hline \multirow{2}{*}{ Placebo } & No & 8 & 36.4 & 7 & 31.8 & 0.750 \\
\cline { 2 - 7 } & Yes & 14 & 63.6 & 15 & 68.2 & \\
\hline \multirow{2}{*}{$\begin{array}{l}\text { Analge- } \\
\text { sic }\end{array}$} & No & 12 & 54.5 & 9 & 40.9 & 0.365 \\
\cline { 2 - 7 } & Yes & 10 & 45.5 & 13 & 59.1 & \\
\hline
\end{tabular}

Post Obturation Records

\begin{tabular}{|l|c|c|c|c|c|c|}
\hline \multirow{2}{*}{ No } & \multicolumn{2}{c|}{$\begin{array}{c}\text { Group A } \\
\text { (intervention) }\end{array}$} & \multicolumn{2}{c|}{$\begin{array}{c}\text { Group B } \\
\text { (Control) }\end{array}$} & \\
\cline { 3 - 7 } & & $\%$ & No & \% & P value & \\
\hline \multirow{2}{*}{ Placebo } & No & 21 & 95.5 & 20 & 90.9 & \multirow{2}{*}{0.550} \\
\cline { 2 - 6 } & Yes & 1 & 4.5 & 2 & 9.1 & \\
\hline
\end{tabular}

Table 4: Frequencies (n), percentages and results of Chi-square $\left(\mathrm{x}^{2}\right)$ for comparing drug intake for the tested groups (Group A: occlusal reduction and Group B: no occlusal reduction).

* Statistically significant ( $\mathrm{p}$-value $\leq 0.05$ ).

\section{Discussion}

Pain is the primary reason that dental patients seek endodontic therapy. For endodontic treatment to be considered successful and accepted readily by the patient and the dentist, it must be firmly associated with the highly efficient relief of pain [28].

Occlusal reduction is one of several strategies that could manage pain and discomfort after root canal treatment $[11,12]$ as well preoperative analgesics, corticosteroid prescription [13-18] and administration of long-acting anesthesia [13].

Despite the positive conclusions of previous studies [12,19-20] on the effect of occlusal reduction on postoperative pain, other studies $[16,21]$ reported that occlusal reduction has no effect on postoperative pain management.

The variation in results of previous studies creates a dilemma for dentists regarding whether they should reduce occlusal con- tacts to prevent pain after root canal treatment or not. Therefore, the purpose of the present study is to evaluate the effect of occlusal reduction on pain after root canal treatment in patients with irreversible pulpitis with symptomatic apical periodontitis.

The present study was designed as a double-blind, parallel design, randomized clinical trial (RCT) to evaluate the effect of occlusal reduction on post- instrumentation and post-obturation pain intensity. Randomized controlled clinical trial is a prospective, analytical, experimental study using primary data generated in the clinical environment. Individuals similar at the beginning are randomly allocated to two or more treatment groups and the outcomes of the groups are compared after sufficient follow-up time.

In this study, 44 mandibular posterior teeth with symptomatic irreversible pulpitis and symptomatic apical periodontitis with no periapical radiographic radiolucency were included because of the higher prevalence of postoperative pain in these cases.

Mandibular posterior teeth usually produce more post-endodontic pain than maxillary ones as the mandible has a dense trabeculae pattern, which causes reduced blood flow and localization of infection leading to delayed healing patterns. Furthermore, preoperatively symptomatic teeth were shown to be significantly associated with postoperative pain $[3,6,8]$.

Several studies stated that the age and gender of the patient have no significant effect on mean pain values. So, males and females with age range from 18 to 50 years old participated in the study.

In the present study, root canals were prepared in a crown-down technique using rotary Revo-S files. In crown-down technique, the bulk of tissue debris and microorganisms are removed before apical instrumentation is commenced, which greatly reduces the risks of extrusion causing periapical inflammation. Therefore, crowndown technique has less postoperative pain than step-back technique.

Revo-S system has great flexibility due to its asymmetrical cross section, snake-like motion. Research reported its decreased apical extrusion of debris and that it can be safely used in curved canals without transportation. 
Watkins., et al. and Oginni., et al. proved that $2.6 \% \mathrm{NaOCl}$ have a good biocompatibility compared to $5.2 \% \mathrm{NaOCl}$ which proved to be the most toxic irrigant, also, they both can reduce bacterial count with the same effectiveness.

The preparation was completed using $2.6 \% \mathrm{NaOCl}$ solution in side-vented irrigation needles to insure least apical extrusion of irrigant. No intracanal medication was used between the 2 visits to avoid the introduction of other variables that might affect postoperative pain. Obturation was done using cold lateral compaction technique as it has less effect on post-obturation pain compared to thermal based obturation techniques.

Studies have shown that AdSeal resin sealer provides good root canal adaptation, apical sealing, biocompatibility, insolubility, radio-opacity and film thickness.

The primary outcome of the present study was to assess incidence and intensity of post-instrumentation and post obturation pain using the modified Visual Analogue Scale (MVAS) during pain rating scale. This scale is statistically the most robust, valid and reliable method of pain assessment. All demographic data, clinical findings and VAS scores obtained from patients, were statistically analyzed.

Participants were instructed to record their pain intensity preoperatively and after $6,12,24$ and 48 hours from the end of endodontic instrumentation as postoperative pain was found to be negligible after 48 hours following root canals preparation. Also, the pain intensity was recorded after 6 and 12 hours from the end of root canal obturation as studies have shown its rise again at 6 hours following root canal obturation as a result of pressure exerted during root canal obturation.

All patients reported moderate to severe pre-operative pain with no significant difference between the two groups in preoperative pain intensity. Statistical analysis of demographic data regarding mean age values, gender distribution and tooth type revealed no significant difference between the two groups. These findings provided a homogenous data which eliminating confounding variables and allowing for a fair comparison of the effect of the intervention (occlusal reduction) in the two groups.

In the present study, there was no significant difference between (occlusal reduction) group and (no occlusal reduction) group in in- cidence of pain $6,12,24$ and 48 hours post-instrumentation and 6 and 12 hours post-obturation. Moreover, statistical analysis revealed no significant difference between the two groups in the median VAS scores of post-instrumentation and post-obturation pain intensity at all follow-up periods. These results are in agreement with some studies $[16,21]$ but in contrast to other studies $[12,19,20]$ who reported that occlusal reduction has a significant effect on reducing postoperative pain. This variability in the results may be attributed to the difference in the inclusion and exclusion criteria, methodology and clinical procedure like use of intra canal medication between the visits as reported in previous studies [20] which could be a significant variable that reduces post-operative pain.

Regarding the median pain score over time in each group (occlusal reduction group and no occlusal reduction group), there was a statistically significant difference comparing preoperative pain with all follow up periods, as pain intensity were changed with time until it reached a maximum decrease 48 hours later in both groups following root canal instrumentation.

The secondary outcome is to assess the incidence of placebo and analgesic intake and number of analgesic tablets taken.

A placebo is a pharmacologically inert substance with no therapeutic effect. It is used to be able to demonstrate that an analgesic is actually effective as it acts by controlling anxiety and it was shown to be effective in high percentage of patients.

After each visit, the patients were given a placebo as an analgesic and were asked to take it in case of moderate to severe pain. If pain persisted after taking the placebo, Ibuprofen $400 \mathrm{mg}$ was prescribed.

NSAIDs (non-steroidal anti-inflammatory drugs) are usually administered as the first analgesic choice subsequent to root canal treatment, among which ibuprofen is the most commonly used medication.

The secondary outcome was to assess the incidence of placebo and analgesic intake and number of analgesic tablets taken. Results showed that the percentage of patients who had taken analgesics in the control group was higher than occlusal reduction group. However, there was no statistical significant difference in the in- 
cidence and number of placebo and analgesic intake between the two groups.

In the present study, it could be concluded that occlusal reduction had no effect on postoperative pain reduction at 6 hours, 12 hours, 24 hours and 48 hours post-instrumentation and at 6 hours and 12 hours post-obturation. There was no significant difference in incidence of analgesic intake between the two groups.

\section{Conclusion}

Within the limitations of this study, we conclude that occlusal reduction in teeth with irreversible pulpitis and with tenderness to percussion had no significant influence on postoperative pain after root canal preparation when compared with teeth with no occlusal reduction. Pain intensity gradually decreases by time following endodontic treatment.

\section{Bibliography}

1. Sathorn C. "The prevalence of postoperative pain and flare-up in single- and multiple-visit endodontic treatment: a systematic review". International Endodontic Journal 10 (2007): 1-9.

2. Siqueira JF Jr., et al. "Incidence of postoperative pain after intracanal procedures based on an antimicrobial strategy". Journal of Endodontics 28.6 (2002): 457-460.

3. Zuckerman O., et al. "Flare-up during endodontic treatment: etiology and management". Refuat Hapeh Vehashinayim 24 (2007): 19-26.

4. Pochapski MT., et al. "Effect of pretreatment dexamethasone on postendodontic pain". Oral Surgery, Oral Medicine, Oral Pathology, Oral Radiology, and Endodontology 108 (2009): 790795.

5. Jalalzadeh SM., et al. "Effect of pretreatment prednisolone on postendodontic pain: a double-blind parallel-randomized clinical trial”. Journal of Endodontics 36 (2010): 978-981.

6. Rosenberg PA. "Clinical strategies for managing endodontic pain”. Journal of Endodontics 3 (2002): 78-92.

7. Rosenberg PA., et al. "The effect of occlusal reduction on pain after endodontic instrumentation". Journal of Endodontics 24 (1998): 492-496.
8. Moore PA and Dunsky JL. "Bupivacaine anesthesia: a clinical trial for endodontic therapy". Oral Surgery, Oral Medicine, Oral Pathology, and Oral Radiology 55 (1983): 176-179.

9. Dunsky JL and Moore PA. "Long-acting local anesthetics: a comparison of bupivacaine and etidocaine in endodontics". Journal of Endodontics 10 (1984): 457-460.

10. Parirokh M., et al. "Effect of bupivacaine on postoperative pain for inferior alveolar nerve block anesthesia after single-visit root canal treatment in teeth with irreversible pulpitis". Journal of Endodontics 38 (2012): 1035-1039.

11. Jostes JL and Holland GR. "The effect of occlusal reduction after canal preparation on patient comfort". Journal of Endodontics 10.1 (1984): 34.

12. Sheikh H., et al. "Comparison of post- instrumentation pain in teeth during root canal treatment with or without occlusal reduction". Pakistan Oral and Dental Journal 35.4 (2015): 699701.

13. Zaman H and Ahmed S. "Effect of occlusal reduction on post instrumentation pain in patients with acute irrversible pulpitis". Pakistan Oral and Dental Journal 36.1 (2016): 119-121.

14. Creech JL., et al. "Effect of occlusal relief on endodontic pain". Journal of the American Dental Association 109 (1984): 64-67.

15. Parirokh M., et al. "Effect of occlusal reduction on postoperative pain in teeth with irreversible pulpitis and mild tenderness to percussion". Journal of Endodontics 39.1 (2013): 1-5.

16. Ashgar S., et al. "Occlusal Reduction Reduces Postoperative Pain After Endodontic Instrumentation". Pakistan Oral and Dental Journal 34.3 (2014): 539-542.

17. Arslan H., et al. "The effect of various occlusal reduction levels on postoperative pain in teeth with symptomatic apical periodontitis using computerized analysis: a prospective, randomized, double-blind study". Clinical Oral Investigations 30.4 (2016): 1-7.

18. Zeidan BM. "Evaluation the Effect of Occlusal Reduction on Postoperative Pain in Teeth with Irreversible Pulpitis and Mild Tenderness to Percussion". Journal of Al Rafidain University College 38 (2016): 128-140. 
19. Raza I., et al. "Effect of occlusal reduction on frequency of postoperative pain relief, following an endodontic instrumentation". Pakistan Oral and Dental Journal 36.4 (2016): 650-653.

20. Rechenberg D., et al. "Pain levels and typical symptoms of acute endodontic infections : a prospective, observational study". BMC Oral Health 16.1 (2016): 1-8.

21. Khan AA., et al. "Measurement of Mechanical Allodynia and Local Anesthetic Efficacy in Patients with Irreversible Pulpitis and Acute Periradicular Periodontitis". Journal of Endodontics 33.7 (2007): 796-799.

Volume 5 Issue 9 September 2021

(C) All rights are reserved by Marian Shenoda Fathy., et al. 